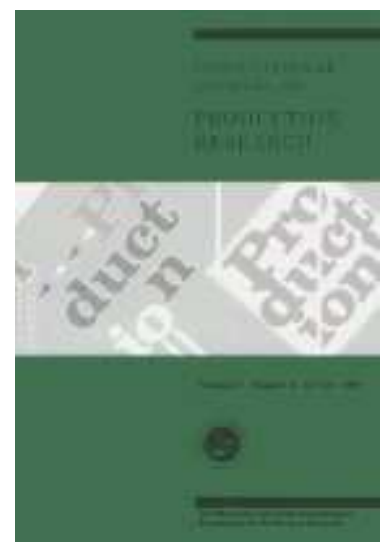

\title{
Assessing Operational Complexity of Manufacturing Systems Based on Statistical Complexity
}

\begin{tabular}{|r|l|}
\hline Journal: & International Journal of Production Research \\
\hline Manuscript ID: & TPRS-2010-IJPR-1144.R1 \\
\hline Manuscript Type: & Original Manuscript \\
\hline Author: & 03-Feb-2011 \\
\hline Complete List of Authors: & $\begin{array}{l}\text { Vrabič, Rok; University of Ljubljana, Department of Control and } \\
\text { Manufacturing Systems } \\
\text { Butala, Peter; University of Ljubljana, Department of Control and } \\
\text { Manufacturing Systems }\end{array}$ \\
\hline Keywords: & $\begin{array}{l}\text { MANUFACTURING CONTROL SYSTEMS, PERFORMANCE MEASURES, } \\
\text { SIMULATION }\end{array}$ \\
\hline Keywords (user): & COMPLEXITY \\
\hline
\end{tabular}

\section{SCHOLARONE"}

Manuscripts 


\section{Assessing Operational Complexity of Manufacturing Systems Based on Statistical Complexity}

Rok Vrabič, Peter Butala

Department of Control and Manufacturing Systems, University of Ljubljana,

Ljubljana, Slovenia

*Corresponding author:

Rok Vrabič,

University of Ljubljana, Faculty of Mechanical Engineering,

Aškerčeva 6,

SI-1000 Ljubljana,

E-mail: rok.vrabic@ffs.uni-lj.si

Phone: +38614771 744

Peter Butala,

University of Ljubljana, Faculty of Mechanical Engineering,

Aškerčeva 6,

SI-1000 Ljubljana,

E-mail: peter.butala@ffs.uni-lj.si

Phone: +38614771 753

\section{Acknowledgements}

This work was partially supported by the Ministry of Higher Education, Science and Technology of the Republic of Slovenia, Grant No. 1000-08-310127, and by the Slovenian Research Agency, Grant No. P2-0270. 


\section{Assessing Operational Complexity of Manufacturing Systems Based on Statistical Complexity}

The aim of this paper is to contribute to complex systems thinking in manufacturing organisations through the development of a metric for operational complexity. Operational complexity is concerned with the temporal aspects of coordination and control in manufacturing systems. Statistical complexity from computational mechanics theory is proposed as the metric. The metric can potentially be used to support decision making by objective assessment of complexity. The properties of the metric are explored through simulation studies. The simulation results confirm that the proposed metric captures the intuitive notion of complexity. It is shown that operational complexity is influenced by internal factors such as system structure, as well as external ones such as demand, and that complexity can be managed through the application of appropriate control methods. A case study is presented that applies the metric to real production data. The case study shows that the global recession had resulted in a decreased operational complexity of outputs.

Keywords: manufacturing systems, complexity, statistical complexity

\section{Introduction}

Modern business organisations are operating in an uncertain and constantly changing environment. Their performance and survival are largely determined by their management of change. Conventional theories of management lack the tools to describe, analyse, and manage the growing complexity, and can, in turn, no longer cope with the issues it gives rise to.

This holds particularly true for manufacturing companies. They are subjected to opportunities and constraints of the global market, cooperating and competing at the same time. While operating in an environment full of disturbances, their main challenge is how to ensure a stable and deterministic delivery of products in spite of the stochastic nature of demand.

A possible approach is offered by interdisciplinary research broadly termed “complexity science," which develops new methods for studying regularities in systems. At its core is an assumption that simple rules and interactions cause complex effects. Although complex systems are frequently unpredictable, they also exhibit 
precise regularities and patterns. These can be used to create strategies that can deal with uncertainty, reduce risk, and expand opportunities of contemporary business (Buchanan, 2004).

Manufacturing companies must deal with two kinds of complexity: the organisation of departments, shop floors, and supply chains on one hand, and the management of the temporal aspects of coordination and control on the other. The former is often termed structural complexity, while operational complexity denotes the latter (Peklenik, 1995). It is hypothesized that by measuring and understanding both, manufacturing organizations will find more efficient ways of operating in the global environment (Wiendahl and Scholtissek, 1994). While structural complexity has already received some attention in literature, operational complexity remains relatively poorly studied.

The principal motivation for the studies of complexity in manufacturing is the search for methods that would allow us to reduce the degree of complexity and make the manufacturing system more productive and predictive (Hon, 2005). In manufacturing control, complexity assessment has practical value for comparison of control methods, for decision analysis, and for the understanding of the control process, especially its complex properties such as self-organization and emergence. The goal is to increase the predictability of the manufacturing system behaviour despite the incompleteness of information, disturbances, and uncertainties inherent to the environment by analyzing the complexity of the control method.

In order to make complexity analysis a practical approach, complexity must be made measurable. Complexity metrics must be calculable and generally applicable. Only as such can they be used for performance measurement and ultimately provide a link between strategy and execution (Melnyk et al., 2004). 
In this paper, statistical complexity from computational mechanics theory is proposed as a metric for the operational complexity of manufacturing systems. Statistical complexity measures the difficulty of process prediction. The abstraction behind statistical complexity is related to symbolic dynamics, wherein a signal is replaced by a sequence of symbols. This sequence is then used as a basis for the reconstruction of an algorithmic automaton. The statistical complexity is the entropy of this automaton's states. This approach provides a practical algorithm for the calculation of the complexity of real systems.

\section{Background}

Manufacturing systems exhibit both fundamental components of complexity: complexity of structure and complexity of operation. Structural complexity can remain unchanged for longer periods of time. On the other hand, operational complexity is continuously influenced by numerous factors such as changes in demand, machine breakdowns, and control methods. Control, in general, provides a lever that can be used to influence the system's behaviour. Operational complexity can therefore only be understood through a closer examination of control.

\subsection{The Manufacturing Control Problem}

Baker (1998) defines manufacturing control as the actuation of a manufacturing system to make products that takes into consideration the present and past observed states of the manufacturing system, as well as economic demand. He proposes a cybernetic model of control wherein the manufacturing system and the control system form a typical feedback loop. While Baker's definition will be adopted throughout this paper, his feedback model fails to capture important properties of manufacturing control systems. 
Firstly, information obtained through the observation of real-life systems is usually incomplete. Furthermore, it is prone to subjective interpretation wherever human subjects are involved. As the toughest problems in manufacturing control are not problems of optimization, but those of a subjective nature, a clearer definition of the role played by human subjects is required. A cybernetic model based on Peklenik’s (1988) manufacturing cybernetics theory is presented in Figure 1.

A manufacturing control system encompasses the whole decision process, including subjects' decisions, leading to the control signals for the manufacturing plant. The information about the inputs and outputs of the plant is acquired by sensory and other processes. The goals are expressed as desired outputs. The control algorithm $\varphi$ describes how control signals are generated based on the goals, the input, and the output information.

The control problem resists formal description, especially when subjects and their decision making are taken into consideration as well. Nevertheless, the problem can be stated as: How to best satisfy the given goals of a manufacturing system, subjected to disturbances, by making decisions based on incomplete data?

To provide an answer to the control problem, we need metrics for the evaluation of solutions. The difficulty in creating such metrics lies in the fact that every manufacturing system has unique properties; general metrics are therefore hard to find. One possibility is to take the approaches from complexity science and apply them to the manufacturing domain. The main hypothesis here is that manufacturing control can be improved if its complexity is measured and analyzed.

\subsection{Information Theory Approaches to Manufacturing Complexity}

As models are specific to a system or phenomenon, a general approach towards complex systems control is difficult to formulate. A promising approach to this 
problem was found in information theory, which allows us to treat different complex systems on an equal footing.

Information theory is well established in complexity science. It provides a well-developed theoretical basis, mathematical definitions, and computational tools. The theory was first established by Shannon (1948). It provides a measure of uncertainty as a function of probabilities, known as the Shannon entropy (1).

$$
H(X)=-\sum_{x \in \chi} P(x) \log _{2}(P(x))
$$

$H(X)$ is a measure of uncertainty or of information about a random variable. It quantifies how much information one is missing when one does not know the value of a random variable.

Entropy is often associated with complexity, because intuitively, the more uncertain an observer is about the observed process, the more complex the process appears. This is studied by Shafiei-Monafred and Jenab (2010) for the case of operations in demand-based manufacturing. They show how human subjects learn to perform a task in less time and then define complexity of manufacturing operations based the learning curve model.

In the manufacturing domain, several complexity metrics are based on the Shannon entropy.

Frizelle and Woodcock (1995) define two types of complexity, static and dynamic complexity, which correspond to the aforementioned structural and operational complexity.

Static complexity is defined as the expected amount of information necessary to describe the state of a manufacturing system. The measure is based on the probability of a resource occupying a state. 
Dynamic complexity is the expected amount of information necessary to describe the state of a system deviating from schedule due to uncertainty. The calculation involves the measurement of the difference between actual performance of the system and the expected figures in the schedule.

Frizelle's complexity metrics have been used and developed further in the work of Efstathiou (1998), Sivadasan (2002), Huaccho Huatuco (2009), and Isik (2010).

ElMaraghy et al. define an entropic complexity metric for manufacturing system configurations (2005). They introduce a complexity coding system to capture the variety and amount of information present in a given manufacturing system and its components.

An entropic definition of complexity is introduced for mixed-model assembly lines by $\mathrm{Hu}$ et al. (2008), and Wang and $\mathrm{Hu}$ (2010), wherein the authors argue that complexity is introduced by product variety. This is also considered by Jenab and Liu (2010) who present a graph-based model for the relative complexity of products.

The above approaches relate complexity to entropy and thus uncertainty. A useful interpretation of this is that entropy measures the amount of information one gains by looking at the actual state of a system. If the system is complex, a lot of information is gained, and vice versa. These methods therefore provide a way of looking at the structural complexity of the system. Even Frizelle's dynamic complexity, which captures the uncertainty of a schedule, is defined for a certain state in time. Operational complexity should, on the other hand, capture how a system evolves in time.

The only approach that explicitly studies temporal aspects of manufacturing systems is presented in (Frizelle and Suhov, 2001), wherein the authors apply the 
Kolmogorov-Sinai entropy to Markovian queues. Complexity is associated with the limiting rate of the entropy production in an observed process, and can be interpreted as the randomness of behaviour in the sense of algorithmic complexity. It has been argued, however, that randomness is not complex, as it easily yields to statistical description. This notion is explored further in the following Section.

\section{Measuring Operational Complexity}

A well-known approach to the complexity assessment of a process through the examination of the sequence of symbols produced by it is the Kolmogorov-Chaitin complexity. It is defined as the length of the shortest program capable of producing the sequence. By this definition, periodic sequences are not complex, as repeating patterns are easily encoded. Random sequences, on the other hand, are considered complex, as they cannot be compressed into a shorter description.

Consider the example of flipping a fair coin. Although the sequence produced by repeating a coin flip is random, coin flipping is intuitively not complex. This is because the intuitive notion of complexity is not associated with the produced sequence but with the underlying process. A coin flipping process is easy to describe in a statistical way: there is a fifty percent chance of heads and a fifty percent chance of tails. Albeit statistical, the description allows for prediction. Even if one cannot know whether the next outcome will be heads or tails, one can make an accurate prediction about the distribution of outcomes over future trials.

In the above sense, complexity is associated with the difficulty of predicting a process, in other words, with the difficulty of discovering the patterns present in the process.

This is readily applicable to the control problem and associated operational complexity. If a manufacturing system's states and outputs are easy to predict, the 
system is not complex. On the other hand, if making predictions is difficult, the system is complex. An operational complexity metric should therefore quantify how difficult it is to predict the future of the system.

Figure 2 presents the complexity curve from the control problem perspective. The complexity curve relates complexity to disorder. Perfectly ordered and perfectly disordered processes have zero complexity. In between, the complexity increases to a point where it becomes incomputable due to the observer's limitations. The area beyond that point is commonly termed "the edge of chaos".

As stated in the control problem definition, one of the goals of control is to make the outputs more deterministic. This, however, is countered by the nature of the inputs, by disturbances, and by the control and manufacturing processes themselves. The more complex these influences, the harder it is to predict the output.

On their way towards becoming outputs (products), inputs (orders, demand) go through a transformation process. At first, they can usually be described statistically accurately with e.g. the Poisson arrival model. Then, as they are scheduled, they transform into manufacturing system states. Their description becomes dependant on the manufacturing system itself. Patterns emerge, depending both on the structure of the manufacturing system as well as on the control method. Complexity increases. Finally, the states are transformed into outputs. Outputs can be considered a coarser description than the states, as different state sequences can lead to the same outputs. Complexity decreases again, as the outputs are in general easier to predict than complete system states.

In light of this, the following Section formally defines an operational complexity metric. 


\subsection{Statistical Complexity}

Statistical complexity attempts to measure the size of the minimum program capable of statistically reproducing the patterns in a data set. It originates in the computational mechanics approach to pattern discovery (Shalizi and Crutchfield, 2001), which has been applied to various fields ranging from molecular systems (Nerukh et al., 2002) to the stock market (Park et al., 2006). The following is a brief illustration of the approach. For a detailed derivation we refer to (Shalizi and Crutchfield, 2001).

\subsubsection{Definition}

A stream of data of infinite length $X$ is split at a chosen point $x_{t}$ into two sequences (2). The sequence up to $x_{t}$ is denoted $\bar{x}$, and the sequence from $x_{t+1}$ on is denoted $\vec{x}$.

$$
X=\underbrace{\ldots, x_{t-2}, x_{t-1}, x_{t}}_{\widetilde{x}}, \underbrace{x_{t+1}, x_{t+2}, \ldots}_{\widetilde{x}}
$$

Causal states $S_{i}$ are defined as sets of $\bar{x}$ for which the probability of an $\vec{x}$ is the same for every $\vec{x}$. The set of causal states is denoted by $S$.

Causal states, together with their transition probabilities, construct an $\varepsilon^{-}$ machine - a minimal model of the process. It has been proven in (Shalizi and Crutchfield, 2001) that causal states are maximally accurate predictors and that their state-to-state transitions are minimally stochastic.

The statistical complexity $C_{\mu}$ is the Shannon entropy (1) over the distribution of causal states (3). $P\left(S_{i}\right)$ denotes the probability of the $\varepsilon$-machine being in state $S_{i}$.

$$
C_{\mu}=-\sum_{S_{i} \in S} P\left(S_{i}\right) \log _{2} P\left(S_{i}\right)
$$

The statistical complexity measures the average amount of historical memory stored in the process. In a complex process, more information about the past is stored internally. Prediction therefore requires more information and is, in turn, more difficult. 
An interesting property of statistical complexity is that it is an upper bound of excess entropy $E$ (Shalizi and Crutchfield, 2001), which is the mutual information of the processes semi-infinite past and semi-infinite future (4). This allows for another interpretation of statistical complexity. The memory needed to perform an optimal prediction is greater or equal to the amount of information that the past provides about the future.

$$
E=I(\bar{x}, \vec{x}) \leq C_{\mu}
$$

The illustration of the $\varepsilon$-machine concept is provided in Figure 3, where $\varepsilon$ machines are shown for completely random and completely deterministic processes.

The random process $(\mathrm{A}$ - e.g. coin-flipping) has the statistical complexity of zero. The deterministic process (B) has the statistical complexity of one bit. This is because only one previous state has to be remembered to optimally predict the one that follows it. The same process can be encoded differently (C) and have a different statistical complexity. Considerations in this regard are analyzed in Section 3.3.

\subsubsection{Calculation}

Statistical complexity can be estimated by considering finite lengths of the symbolic sequence under observation. Shalizi C.R. and Shalizi K.L. (2004) present a CausalState Splitting Reconstruction (CSSR) algorithm, which is able to reconstruct the $\varepsilon$ machine of the process. Although the approach offers great practicality, there are also some limitations to it.

The maximum history length considered, $L_{\max }$, is an adjustable parameter. It is shown that for periodic processes, the lower bound of $L_{\max }$ is the period. The upper bound is limited by adequate sampling to the order of the logarithm of the whole sequence length, $\log (N)$. 


\subsection{Observation Levels}

In complex systems, the observer plays a crucial role. Emergence, as the defining phenomenon of complex systems, has no meaning without an observer. Because emergence is the occurrence of something new, it has to be specified for whom it is new.

Consider an example of a manufacturing system. Machine operators, production managers, and business managers all observe the same system. Their views are partial and acquired on different structural and temporal scales. The operators mainly deal with single elements of the system. The production managers are concerned with the state of the system as a whole. Lastly, the business managers are mostly interested in system outputs. Temporal scales vary as well, from seconds at the operator level to days at the business level.

Resolution of observation is subjective, giving rise to two questions: Can different levels of observation of the same system co-exist, and, if they can, how to determine the optimal level of observation and optimal type of description?

Shalizi and Moore (2003) argue that different levels of observation of the same system can co-exist from the perspective of computational mechanics theory. Comparing a fine and a coarse-grained description, each may have its own, coherent causal account, which gives legitimacy to using different resolutions and time scales in describing the same process.

Shalizi (2001) furthermore suggests that the optimal level is determinable and defines it as the level at which the ratio of the extent of predictability to the difficulty of prediction is highest (Prokopenko et al., 2009). This metric has been termed "efficiency of prediction", $e$ (5), and is defined as the quotient of excess entropy (4) and statistical complexity (3). 


$$
e=\frac{E}{C_{\mu}}=\frac{\text { How much can be predicted. }}{\text { How difficult it is to predict. }}
$$

It can be interpreted as the fraction of historical memory stored in the process, which is useful in telling us about the future. Given two possible observation levels, the one with higher efficiency of prediction should be chosen.

These concepts are studied in the following Section, where the operational complexity metric is applied to a simulated system. The simulation results are observed on different levels and with different encodings.

\section{Simulation Experiments}

Three groups of simulation experiments are carried out to analyze the properties of the proposed operational complexity metric. The first group studies the effect of the control algorithm on complexity. The second group shows how structural complexity affects the operational complexity and the implications of observing this on different levels. Finally, the third group studies the introduction of periodicity into the control algorithm.

\subsection{Problem Formulation}

A simulation model of a simple make-to-order manufacturing system is developed. The system consists of two machines, both capable of processing two types of jobs. Jobs have fixed processing times and due times, relative to arrival times. Job processing cannot be interrupted. The Poisson arrival model is used for job arrival times. For successful completion in due time, the system receives a fixed award. If a job is not completed in its due time, the penalty, which is subtracted from the award, increases linearly with time. Job details are outlined in Table 1.

$\mathrm{J} 1$ are shorter jobs with a minor award. J2 are lengthier and carry a significantly greater award. As such, they implicitly have a higher priority. 
The goal of control is to maximize the reward. In this case, a genetic algorithm approach is chosen for the control algorithm for several reasons. Firstly, the problem is non-deterministic, because the arrival times are generated randomly. It is more important for the algorithm to find a good solution in a short period of time than for it to find the optimal solution, as the optimum may change with every new job arrival anyway. Secondly, modifying the parameters of the genetic algorithm allows us to test the changes in control algorithms. The complexity measure can then be evaluated with regards to the algorithm parameters. Lastly, the specified goal is easily encoded into a fitness function.

For the genetic algorithm, a direct approach with a job-based encoding is used (Hongze et al., 2009). The crossover is position-based. Swap mutation is used (Cheng et al., 1996). The genetic algorithm takes the jobs that had not been processed yet and schedules them to machines. The fitness function of the algorithm maps a schedule encoding to its calculated received reward. The schedules specify job orders for each machine. Jobs are processed sequentially with zero in-between time.

The simulated control problem is presented in Figure 4.

For the analysis, inputs, states, and outputs are observed at different levels and with different encodings $\left(\mathrm{O}_{\mathrm{X}}, \mathrm{O}_{\mathrm{S}}\right.$, and $\mathrm{O}_{\mathrm{Y}}$ respectively). The observations are made on each step of the simulation.

Inputs are encoded using the job type. The encoding process is as follows. If no job arrives, the inputs are encoded to be "0". Inputs are encoded to be " 1 " and "2" when jobs of type $\mathrm{J} 1$ and $\mathrm{J} 2$ arrive, respectively. If jobs of both types arrive on the same simulation step, the inputs are encoded to be " 3 ".

Similarly, the states are encoded in three different ways. "Full states" by using machine ID and job type, "states" by using machine ID and whether the machine is 
processing a job or is idle, and "trivial states" by observing whether any of the machines is processing a job or not.

The outputs are encoded in two different ways: "outputs" by using the job type and "trivial outputs" by only considering whether something was produced or not.

Three groups of experiments are carried out. In each case, the experiment ends after 1000000 simulation steps and is repeated 10 times. Statistical complexity is evaluated using the CSSR algorithm.

\subsection{Standard Configuration with Different Algorithm Properties}

In this group of experiments, the effects of the control algorithm on complexity are analyzed. In order to do this, genetic algorithm scheduling is compared to random scheduling. In the genetic algorithm case, population size is set to 20 , mutation rate is set to 0.05 , and the number of generations is fixed at 5 . In the case of random scheduling, schedules are generated randomly. Both algorithms produce a new schedule when a new job arrives.

Results are shown in Table 2.

The complexity of randomly generated inputs, encoded in the same way as outputs, was calculated to be 0 in both cases. The result is trivial, but confirms complete randomness is adequately recognized by the calculation method.

Although the inputs are random and thus easy to predict, the outputs are not, even in the case of random scheduling. This shows that some inherent structure is present in the system.

In the presented scenario, output patterns are a consequence of rules implicitly formed by the system and job configuration. In the example, two outputs from the same machine cannot be fewer than 12 simulation steps apart, because 12 is the 
processing time for the shortest possible job. Rules like this limit the possible output sequences and are the reason behind the observed output pattern.

When the genetic algorithm is introduced, the complexity of outputs and states decreases. In other words, they become easier to predict. This is in agreement with the intuitive notion that control increases the predictability of the outputs and decreases the effort needed to make the prediction.

An interesting result is that when the states are observed on the "trivial states" level, the complexity increases when control is introduced. Both configurations produce the same automaton configuration, depicted in Figure 5.

The difference is that when random scheduling is used, the probability of the system being in state 1 (idle) decreases and the probability of state 14 (continuous work) increases. Higher probability of state 14 decreases the uncertainty of the automaton state, subsequently giving lower complexity.

Figure 5 also clearly shows the pattern discovered by the method. The left part of the automaton represents processing of a single $\mathrm{J} 1$ job. Processing of $\mathrm{J} 2$ jobs takes place in state 14 - continuous work.

The case of trivial state encoding also shows that by choosing a level of observation that is too coarse, the complexity is not captured adequately. Efficiencies of prediction, presented in Table 2 , show that prediction is significantly less efficient on the trivial states level. Consequently, this level is less suitable for observation, as it gives rise to no new emergent patterns, which would result in higher efficiency of prediction.

The contribution of this set of experiments is twofold. Firstly, controlling regularities by means of a control algorithm can be used to manage the complexity. In the case presented, outputs and states become easier to predict. Secondly, however, 
this has to be observed on an appropriate level, as a coarse-grained projection of the observation can have different complexity properties. Efficiency of prediction can be used to guide the observation level selection.

\subsection{Configuration with Three Machines}

This group of experiments is designed to test how the system structure influences operational complexity. Two more scenarios are devised in addition to the original one with two machines $(2 \mathrm{M})$. The second scenario (3ME) uses three machines, while inputs remain the same as in the $2 \mathrm{M}$ scenario. In the third scenario $(3 \mathrm{MH})$, three machines are used as well. The average job inter-arrival times are modified, so that jobs arrive proportionally more frequently for three machines as they do for two. The scenarios are compared in Figure 6.

The results show that output complexity is mainly influenced by input job configuration, regardless of encoding. In $2 \mathrm{M}$ and $3 \mathrm{ME}$ cases, in which input job configuration is the same, output complexity does not differ significantly. In the $3 \mathrm{MH}$ case, where more jobs arrive per time unit, the output complexity rises.

The state complexity, on the other hand, is mostly influenced by the number of machines. This is understandable, as more machines yield a bigger state space.

The trivial states level again behaves differently from the states and full states levels. As in the previous case, the automaton is the same in all three scenarios (Figure 5). The complexity increases in the 3ME case because the probability of being idle increases, and drops in the $3 \mathrm{MH}$ case because the probability drops. This is a consequence of the fact that there is a greater probability that at least one machine is processing a job at all times in the $3 \mathrm{MH}$ case.

Another interpretation is provided by the complexity curves in Figure 6 b). The inputs exhibit complexity of zero in all cases. As they transform into states, the 
complexity rises depending on the system structure. The output complexity is then mainly determined by the input configuration.

This group of experiments suggests that complexity should be studied by simultaneously observing the system at different levels. Aspects of how operational complexity is influenced by structural complexity can, for example, be quantified by observing the system states. Properties of the inputs, conversely, can be quantified by observing the outputs. The complete view on operational complexity can only be obtained by observing both.

\subsection{Configuration with Periodic Control}

The main question for this group of experiments is whether complexity can be decreased by introducing periodicity into the control algorithm, as hypothesized by Suh (2005).

Periodic control was simulated by running the genetic algorithm periodically. Four experiments were carried out with periods of one (every step), two, four, and eight steps. The algorithm processes all pending jobs only at the beginning of each period.

The results in Figure 7 show that output complexity does decrease, but does not do so significantly. In relation to the previous group of experiments, this is speculated to be a consequence of the fact that output operational complexity is mostly influenced by input configuration.

Conversely, the state complexity does decrease significantly, but only to a certain point. The decrease of complexity is a consequence of the fact that jobs are packed one after another more closely. This results in longer periods of continuous work followed by longer periods of the system being idle. The future states consequently become easier to predict. 
Periodic control is hereby shown to lower the operational complexity of system states. As such, it can be used as a countermeasure to the growth of complexity due to structure, which was shown in the previous Subsection.

\section{Case Study}

The practicality of the approach is shown in a case study based on real data acquired from a manufacturing company. The production is serial, running 24 hours a day in three shifts.

Data was acquired for five manufacturing centres over a period of 24 months, from October 2007 to October 2009. The data consists of times, at which the outputs were produced, along with several process parameters. In the case study, the productivity data is analyzed to assess the complexity of the outputs.

The method of encoding the data is adopted from (Nerukh et al., 2002).

Firstly, the number of pieces produced per hour is calculated for the whole period. Quantities are encoded with bins of size 20. This means that for zero produced pieces, the value is set to 0 , for $1-20$, the value is set to 1 , etc. The complexity for each month is calculated using a three month window in order to achieve the required data length. CSSR algorithm is used to reconstruct the automatons. The length of the analyzed string is 16 .

\subsection{Results and Discussion}

Figure 8 shows complexity and production rate for the period between November 2007 and August 2009.

The operational complexity of outputs remains high, at approximately 11 bits, throughout 2008. In December 2008 and January 2009, no pieces were produced for a period of three weeks as a consequence of the holiday season. The complexity, accordingly, sharply drops to less than 3 bits in this period. 
In 2009, complexity increases again but never reaches the heights of 2008 and fluctuates around approximately 9 bits. This remains true even in July 2009, when slightly more pieces were produced than in August 2008 (marked on Figure 8).

In light of the findings of simulation studies, lower output complexity in 2009 is a direct consequence of lower demand, which is itself a consequence of the global recession. Lower demand also influences the complexity indirectly. In 2009, the management introduced free weekends in order to cope with the situation. Thusly, periodicity was implicitly introduced into control. As shown by the simulation studies, this can contribute to the complexity drop.

The results make it clear that there is an intrinsic connection between complexity and the production rate. In most cases, an increased production rate means a higher complexity as well. Some deviations from this occur in July and August 2008 and July and August 2009. In August, the production rate drops each year because of vacations. Complexity, interestingly, remains the same in both years.

\section{Conclusions}

Complexity has been identified as a ubiquitous property of manufacturing systems, one that has to be embraced by complex systems thinking rather than dismissed. It has been hypothesized that our understanding of complexity must be expanded, and that it can be expanded through the development of complexity metrics.

This paper presents a unique view on operational complexity in manufacturing control. Complexity is associated with the difficulty of prediction. Statistical complexity from computational mechanics is proposed as a complexity metric. The metric offers a practical approach to complexity assessment based on a strong information theory background. As such, it allows for an easy implementation and a clear interpretation. 
The properties of the metric are explored in the manufacturing context using simulation studies which highlight the limitations associated with the choice of the observation level. The metric is found to be both subjective and objective. It is determined by the subjective choice of the observation level, but is calculated deterministically based on the observed time series. This allows for a comparison of different systems when they are observed at the same level.

Several implications of the choice of the observation level are outlined. It is suggested complexity should be studied by simultaneously observing the system at different levels. A complete picture of complexity can therefore only be obtained by merging the partial views of different management levels. Efficiency of prediction can be used as a metric that guides the selection of observation levels.

Introduction of periodicity into control is shown to lower the complexity of manufacturing plant states and is suggested to be used as a countermeasure to the growth of complexity due to growth of structure, e.g. with the introduction of new machines.

Practicality of the approach is confirmed by the case study, wherein the operational complexity of outputs is calculated for real data obtained from serial production. The case study uses an established method to encode the data. The results show a drop in complexity as a consequence of the drop in demand in 2009 , which was a reaction to the global recession. This supports the hypothesis that complexity should not be decreased regardless of the cost, but should instead be properly managed.

Although the case study gives some insight into complexity, further studies are needed to build a causal relation between complexity and the events that influence it. Only then could the root causes to specific problems be identified using the proposed 
metric. Future work will focus on exploring the relations between product complexity, process complexity, and manufacturing system configurations complexity in regards to the operational complexity. Other possibilities for future work include development of a general method for assessing complexity in manufacturing systems and a further exploration of the impacts of the levels of observation.

\section{References}

Baker, A.D., 1998. A Survey of Factory Control Algorithms that can be Implemented in a Multi-agent Heterarchy: Dispatching, Scheduling, and Pull. Journal of Manufacturing Systems, 17 (4), 297-320.

Buchanan, M., 2004. Power Laws \& the New Science of Complexity Management. strategy+business, Spring 2004 (34).

Cheng, R., Gen, M. and Tsujimura, Y., 1996. A Tutorial Survey of Job-shop

Scheduling Problems Using Genetic Algorithms, Part I: Representation. Computers \& Industrial Engineering, 30 (4), 983-997.

Efstathiou, J., Calinescu, A. and Blackburn, G., 2002. A Web-based Expert System to Assess the Complexity of Manufacturing Organizations. Robotics and ComputerIntegrated Manufacturing, 18 (3-4), 305-311.

ElMaraghy, H., Kuzgunkaya, O. and Urbanic, R., 2005. Manufacturing Systems Configuration Complexity. CIRP Annals - Manufacturing Technology, 54 (1), 445450. 
Frizelle, G. and Suhov, Y.M., 2001. An Entropic Measurement of Queueing

Behaviour in a Class of Manufacturing Operations. Proceedings: Mathematical,

Physical and Engineering Sciences, 457 (2011), 1579-1601.

Frizelle, G. and Woodcock, E., 1995. Measuring Complexity as an Aid to Developing Operational Strategy. International Journal of Operations \& Production Management, 15 (5), 26-39.

Hon, K., 2005. Performance and Evaluation of Manufacturing Systems. CIRP Annals - Manufacturing Technology, 54 (2), 139-154.

Hongze, Q., Wanli, Z. and Hailong, W., 2009. A Genetic Algorithm-Based Approach to Flexible Job-Shop Scheduling Problem. Proceedings: ICNC '09, 81-85.

Hu, S., Zhu, X., Wang, H., Koren, Y., 2008. Product Variety and Manufacturing Complexity in Assembly Systems and Supply Chains. CIRP Annals - Manufacturing Technology, 57 (1), 45-48.

Huaccho Huatuco, L., Efstathiou, J., Calinescu, A., Sivadasan, S. and Kariuki, S., 2009. Comparing the impact of different rescheduling strategies on the entropicrelated complexity of manufacturing systems. International Journal of Production Research, 47 (15), 4305-4325.

Isik, F., 2010. An entropy-based approach for measuring complexity in supply chains. International Journal of Production Research, 48 (12), 3681-3696. 
Jenab, K., Liu, D., 2010. A graph-based model for manufacturing complexity.

International Journal of Production Research, 48 (11), 3383-3392.

Melnyk, S.A., Stewart, D.M. and Swink, M., 2004. Metrics and Performance

Measurement in Operations Management: Dealing with the Metrics Maze. Journal of Operations Management, 22 (3), 209-218.

Nerukh, D., Karvounis, G. and Glen, R.C., 2002. Complexity of Classical Dynamics of Molecular Systems. I. Methodology. J. Chem. Phys., 117 (21), 9611-9617.

Park, J.B., Lee, J.W., Yang, J., Jo, H. and Moon, H., 2006. Complexity Analysis of the Stock Market. Physica A, 379 (1), 179-187.

Peklenik, J., 1988. Fertigungskybernetik, Eine neue wissenschaftliche Disziplin für die Produktionstechnik. Festvortrag anlässlich der Verleihung des Georg-Schlesinger Preises 1988 des Landes Berlin.

Peklenik, J., 1995. Complexity in Manufacturing Systems. CIRP Journal of Manufacturing Systems, 24 (1), 17-25.

Prokopenko, M., Boschetti, F. and Ryan, A.J., 2009. An Information-theoretic Primer on Complexity, Self-organization, and Emergence. Complexity, 15 (1), 11-28. 


\author{
Shafiei-Monafred, S., Jenab, K., 2010. Complexity analysis of an operation in \\ demand-based manufacturing. International Journal of Production Research, First \\ published on: 21 December 2010 (iFirst).
}

Shalizi, C.R., 2001. Causal Architecture, Complexity and Self-organization in Time Series and Cellular Automata. Thesis (PhD), University of Michigan.

Shalizi, C.R. and Crutchfield, J.P., 2001. Computational Mechanics: Pattern and Prediction, Structure and Simplicity. Journal of Statistical Physics, 104 (3-4), 817879.

Shalizi, C.R. and Moore, C., 2003. What Is a Macrostate? Subjective Observations and Objective Dynamics. arXiv, cond-mat/0303625.

Shalizi, C.R. and Shalizi, K.L., 2004. Blind Construction of Optimal Nonlinear Recursive Predictors for Discrete Sequences. Proceedings of UAI 2004, 504-511.

Shannon, C., 1948. A Mathematical Theory of Communication. CSLI Publications.

Sivadasan, S., Efstathiou, J., Frizelle, G., Shirazi, R. and Calinescu, A., 2002. An Information-theoretic Methodology for Measuring the Operational Complexity of Supplier-Customer Systems. International Journal of Operations \& Production Management, 22 (1), 80-102.

Suh, N.P., 2005. Complexity in Engineering. CIRP Annals - Manufacturing Technology, 54 (2), 46-63. 
Wiendahl, H. and Scholtissek, P., 1994. Management and Control of Complexity in Manufacturing. CIRP Annals - Manufacturing Technology, 43 (2), 533-540.

Wang, H., Hu, S., 2010. Manufacturing Complexity in Assembly Systems with Hybrid Configurations and its Impact on Throughput. CIRP Annals - Manufacturing Technology, 59 (1), 53-56. 


\begin{tabular}{|l|l|l|l|l|l|}
\hline $\begin{array}{l}\text { Job } \\
\text { type }\end{array}$ & $\begin{array}{l}\text { Inter-arrival } \\
\text { time [steps] }\end{array}$ & $\begin{array}{l}\text { Processing } \\
\text { time [steps] }\end{array}$ & $\begin{array}{l}\text { Due time relative } \\
\text { to arrival time [steps] }\end{array}$ & Award & $\begin{array}{l}\text { Penalty } \\
\left.\text { rate [step }{ }^{-1}\right]\end{array}$ \\
\hline J1 & 14 & 12 & 30 & 3 & 1 \\
\hline J2 & 50 & 32 & 40 & 50 & 10 \\
\hline
\end{tabular}

Table 1: Job details 


\begin{tabular}{|l|l|l|l|l|}
\hline CSSR, L=20, average values & $\begin{array}{l}\mathrm{C}_{\mu, \text { input }} \\
{[\text { bits] }}\end{array}$ & $\begin{array}{l}\mathrm{C}_{\mu, \text { output }} \\
{[\text { bits }]}\end{array}$ & $\begin{array}{l}\mathrm{C}_{\mu, \text { state }} \\
{[\mathrm{bits}]}\end{array}$ & $\begin{array}{l}\mathrm{C}_{\mu, \text { trivial state }} \\
{[\text { bits }]}\end{array}$ \\
\hline Random & 0 & 7.68 & 8.14 & 0.93 \\
\hline Genetic algorithm & 0 & 7.07 & 7.44 & 1.39 \\
\hline $\begin{array}{l}\text { Efficiency of prediction } \\
\text { on the observation level }\end{array}$ & $0 \%$ & $96.2 \%$ & $97.3 \%$ & $68.4 \%$ \\
\hline
\end{tabular}

Table 2: Comparison of random and genetic algorithm scheduling from the perspective of statistical complexity 
Figure 1: Manufacturing cybernetics view on the manufacturing control problem.

Figure 2: The complexity curve from the control problem perspective.

Figure 3: $\varepsilon$-machines for a completely random process, a deterministic process, and the same deterministic process, but with a different encoding.

Figure 4: The simulated problem postulated as a control problem.

Figure 5: $\varepsilon$-machine for trivial states - genetic algorithm scheduling.

Figure 6: Average statistical complexity of the $2 \mathrm{M}, 3 \mathrm{ME}$ and $3 \mathrm{MH}$ configurations, observed on different levels.

Figure 7: Statistical complexity with regards to control period.

Figure 8: Statistical complexity and productivity of the analysed data. 


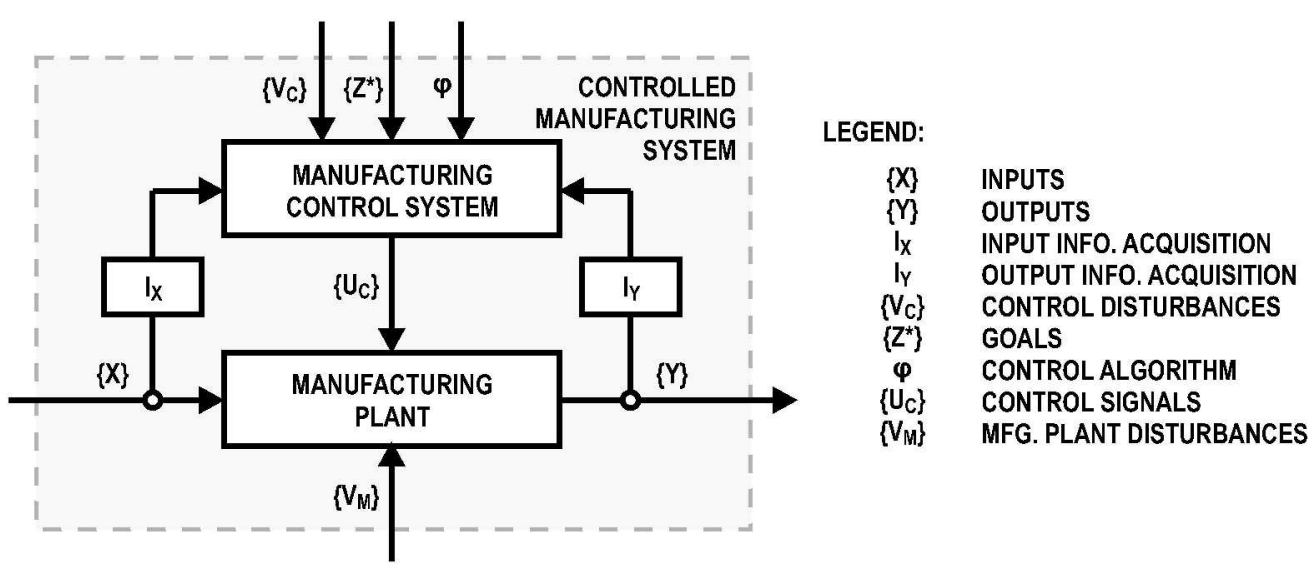

Manufacturing cybernetics view on the manufacturing control problem. $132 \times 55 \mathrm{~mm}(600 \times 600 \mathrm{DPI})$ 


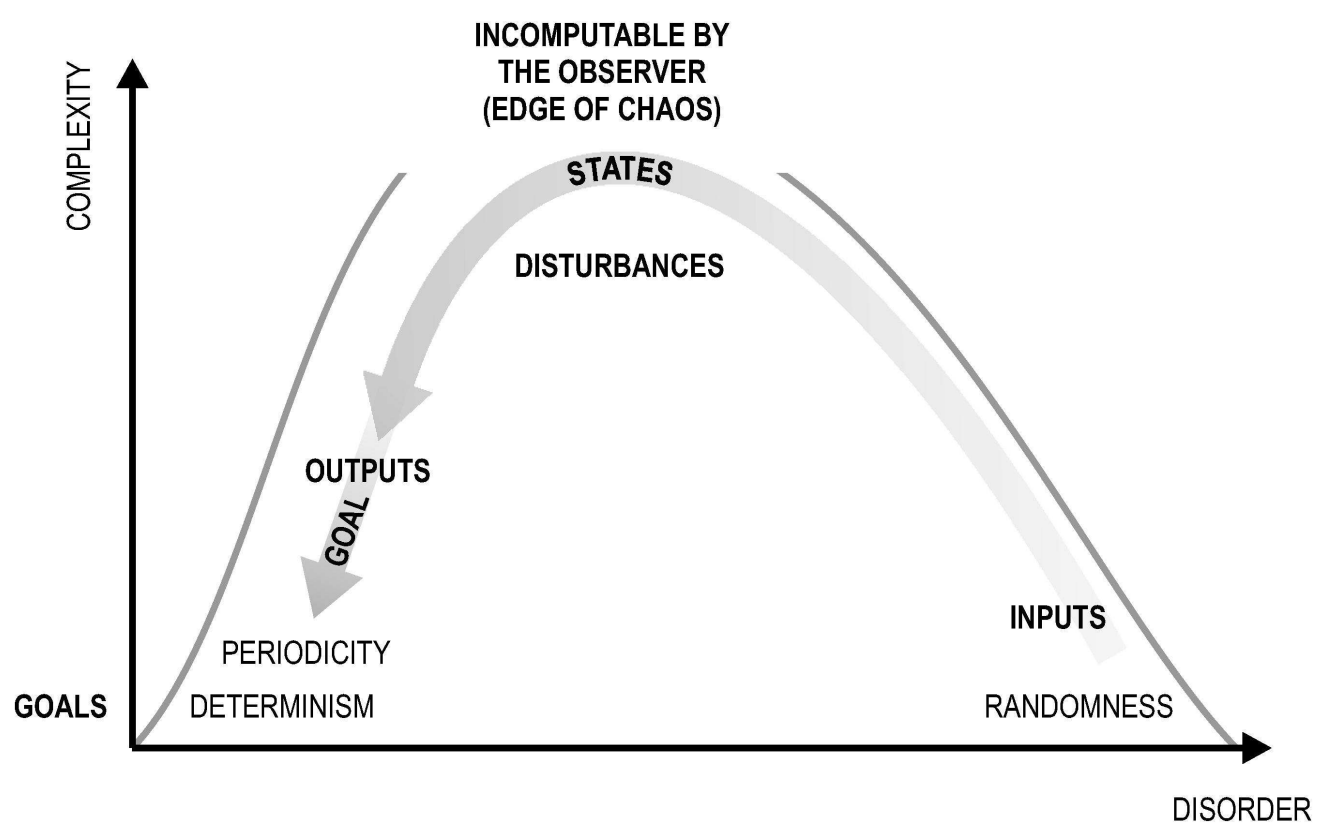

The complexity curve from the control problem perspective. $113 \times 70 \mathrm{~mm}(600 \times 600 \mathrm{DPI})$ 
TYPE

$\varepsilon-M A C H I N E$

PRODUCED SEQUENCE

STATISTICAL COMPLEXITY

A) RANDOM

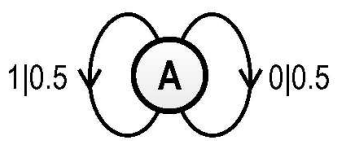

0101100110110111...

0 


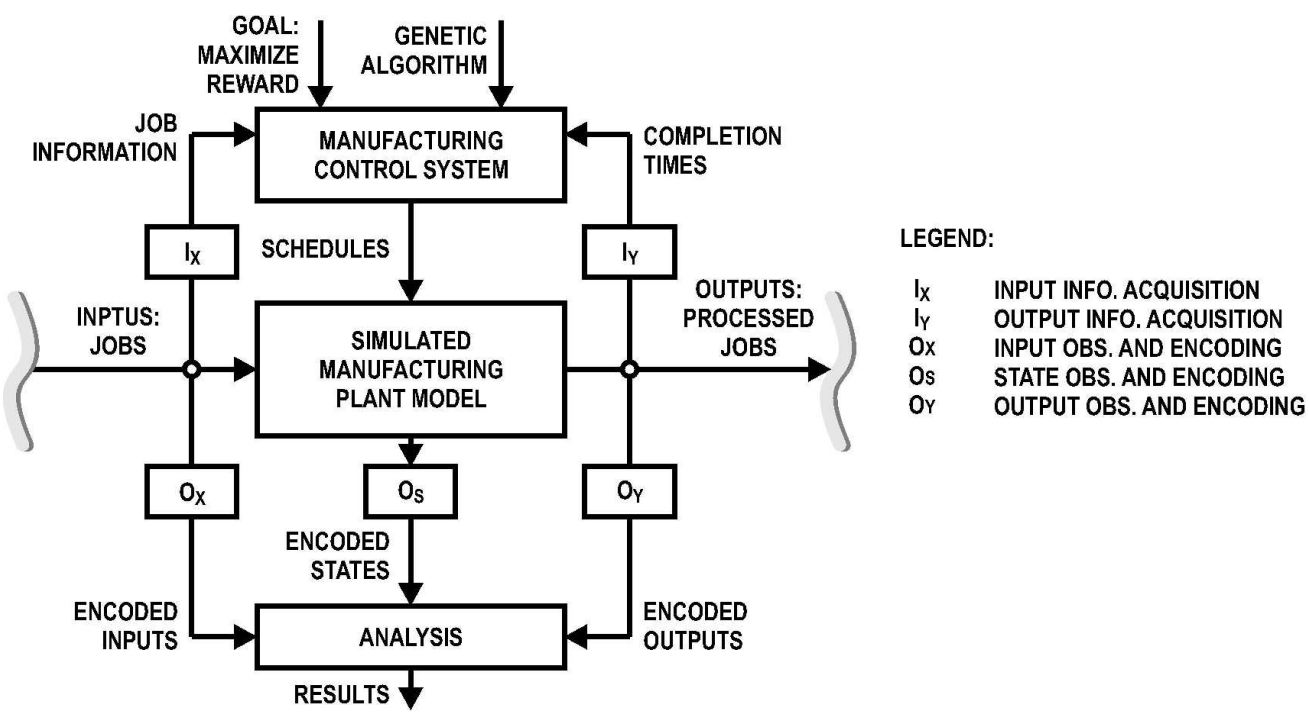

The simulated problem postulated as a control problem. $144 \times 77 \mathrm{~mm}(600 \times 600 \mathrm{DPI})$ 


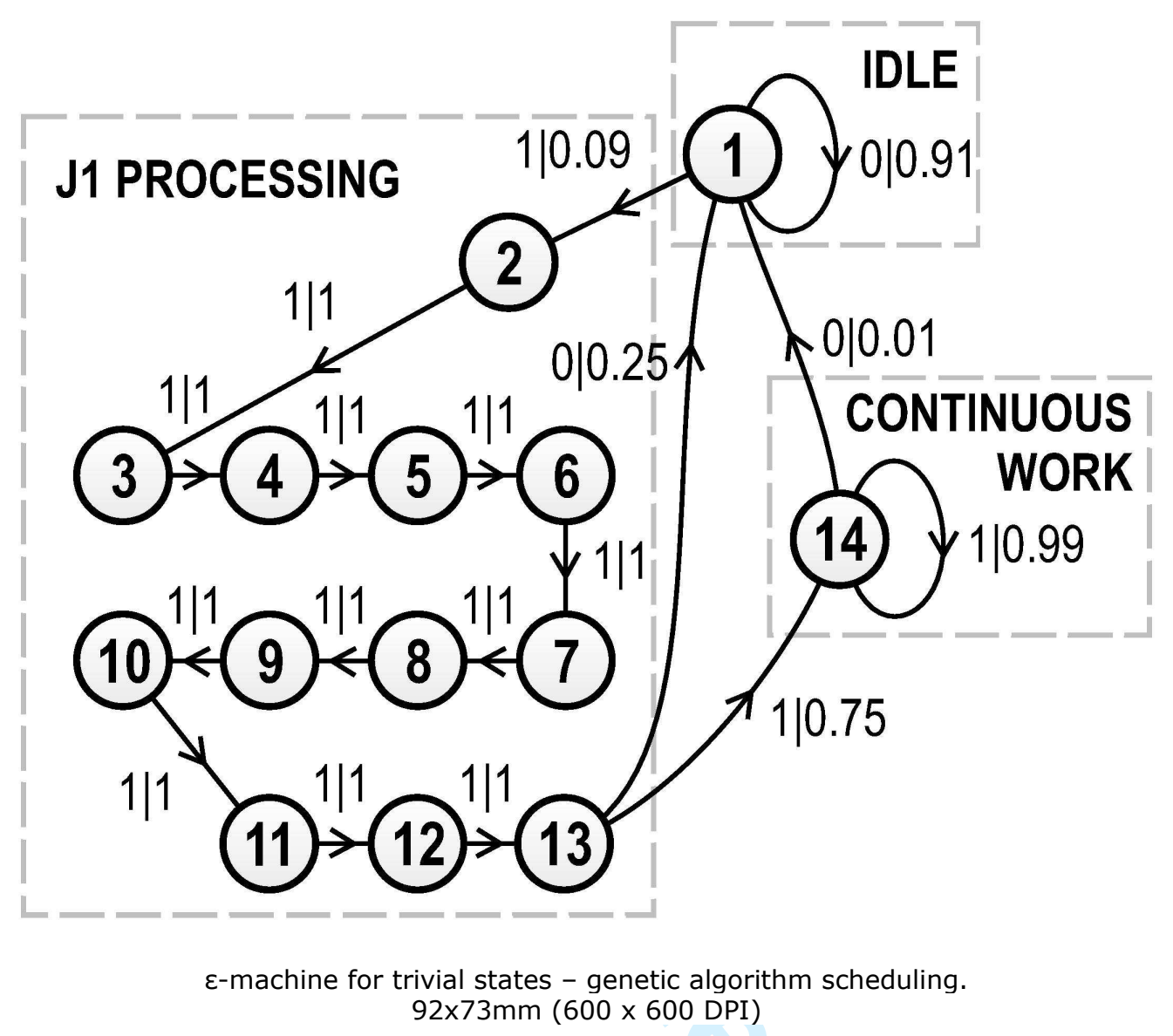

http://mc.manuscriptcentral.com/tprs Email: ijpr@lboro.ac.uk 
a) Statistical complexity $(L=16)$

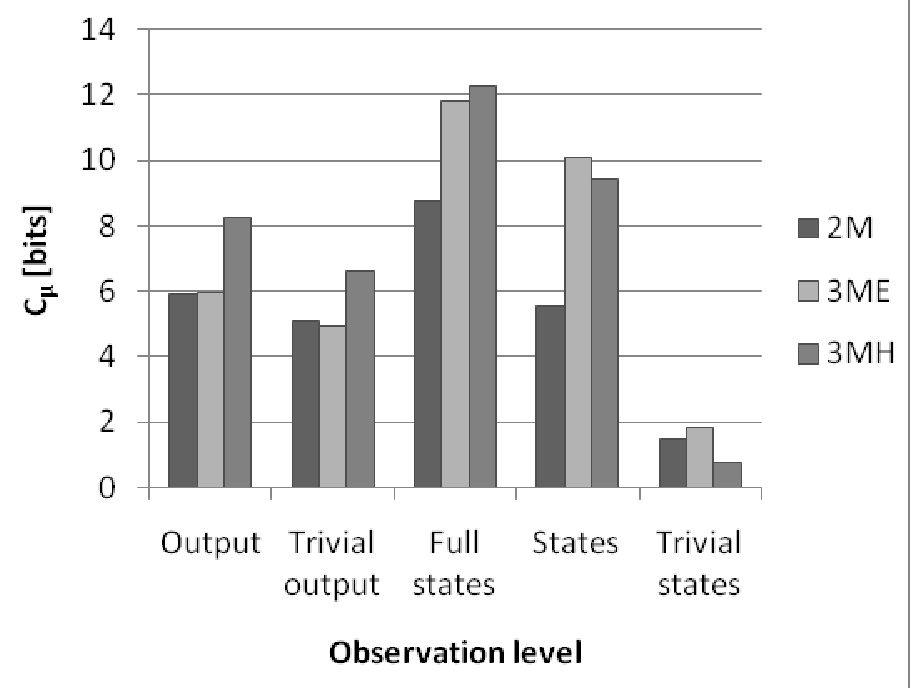

b) Complexity curves (L=16)

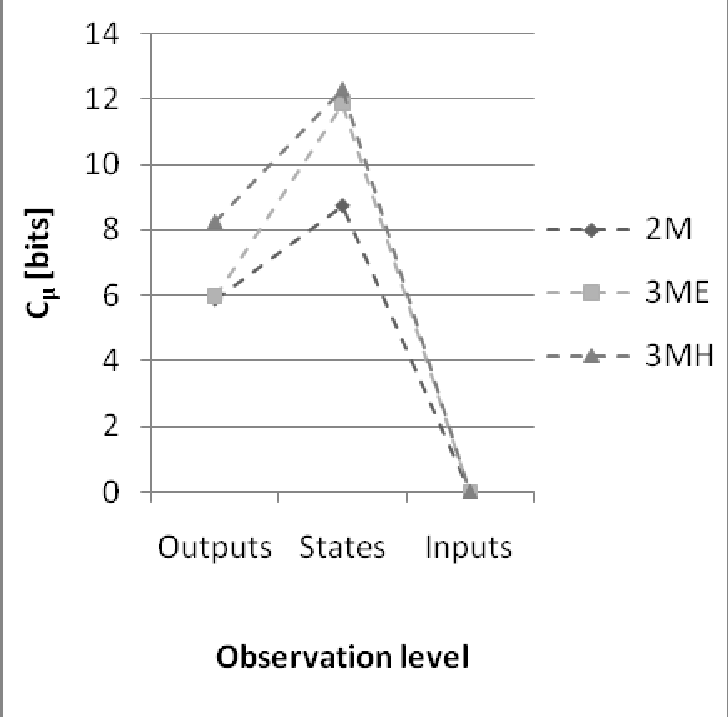




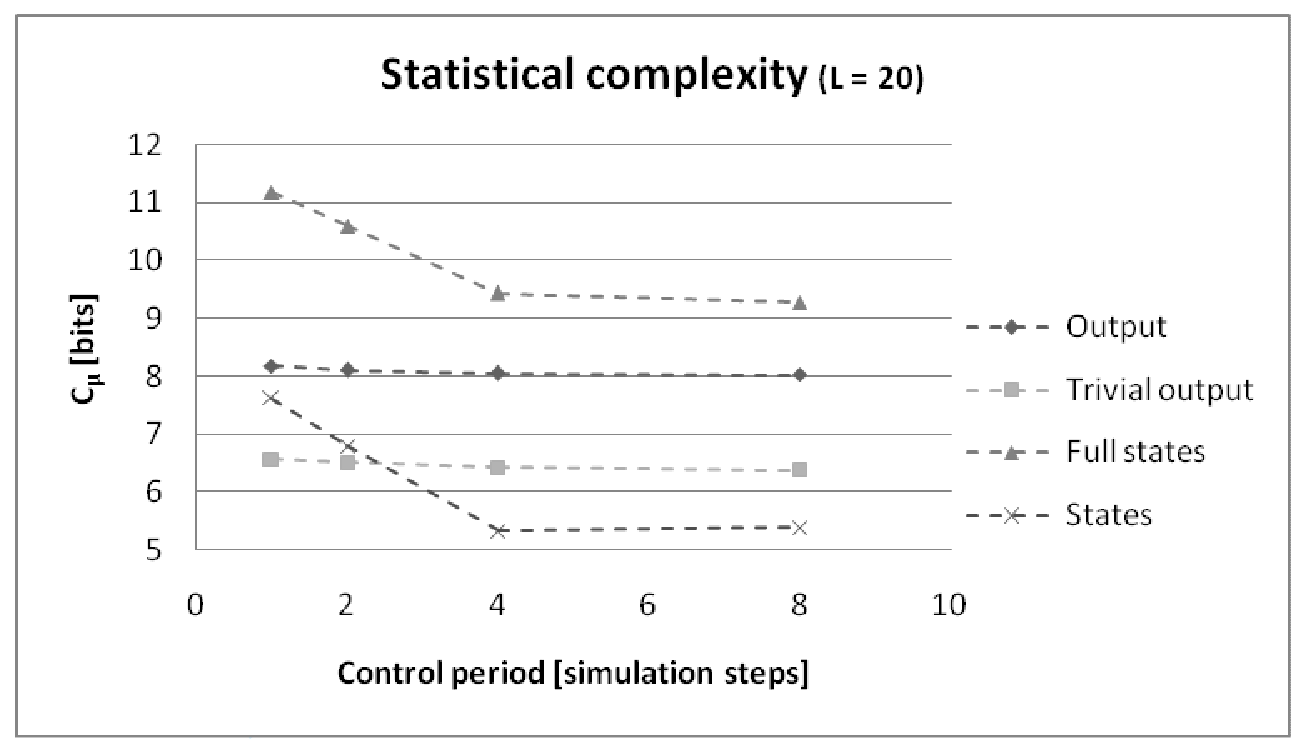




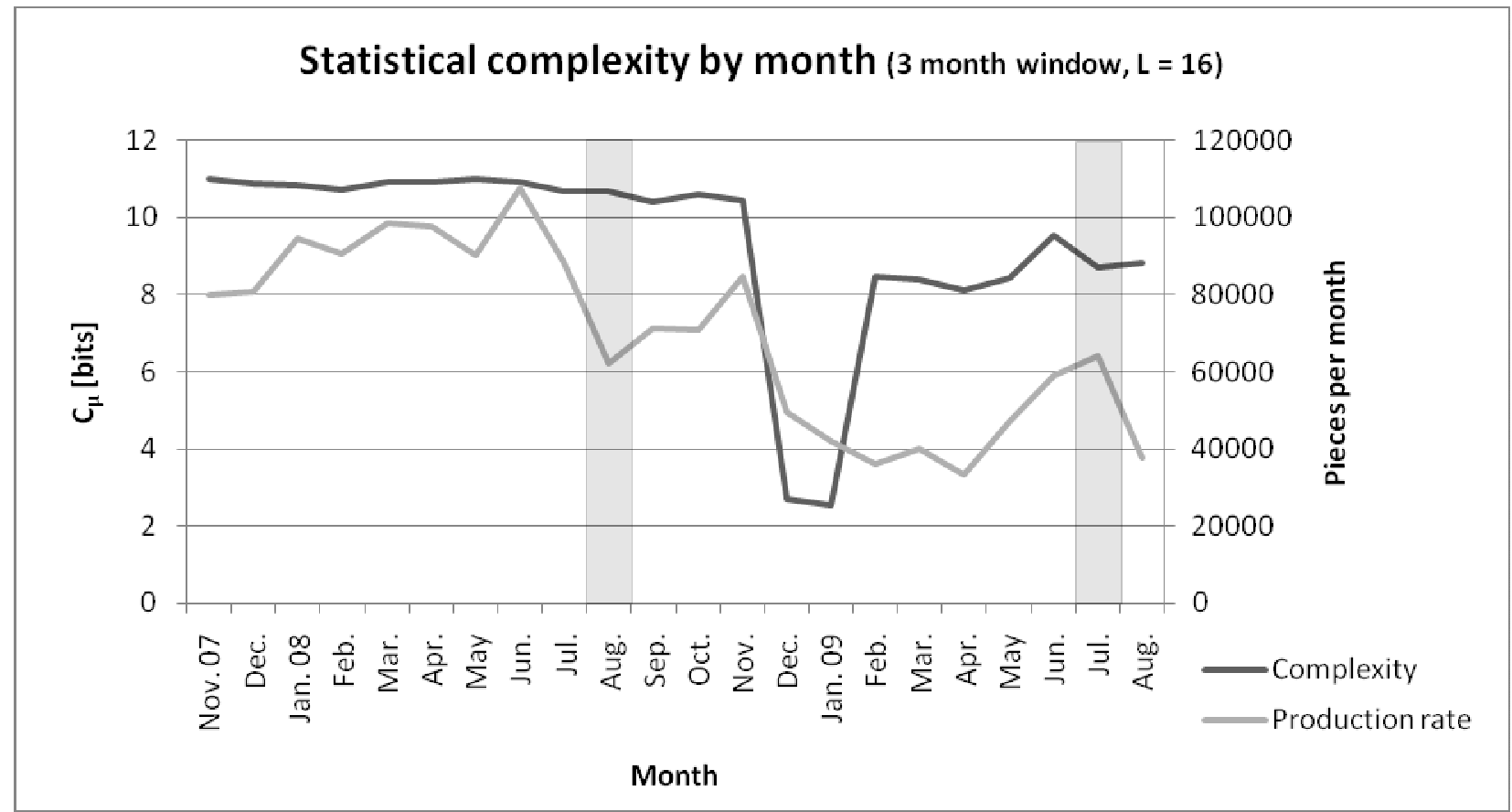

